\title{
Importance-Driven Feature Enhancement in Volume Visualization
}

\author{
Ivan Viola, Armin Kanitsar, and M. Eduard Gröller, Member, IEEE
}

(Invited Paper)

\begin{abstract}
This paper introduces importance-driven volume rendering as a novel technique for automatic focus and context display of volumetric data. Our technique is a generalization of cut-away views, which - depending on the viewpoint or feature - remove or suppress less important parts of a scene to reveal more important underlying information. We automatize and apply this idea to volumetric data.

Each part of the volumetric data is assigned an object importance which encodes visibility priority. This property determines which structures should be readily discernible and which structures are less important. In those image regions, where an object occludes more important structures it is displayed more sparsely than in those areas where no occlusion occurs. Thus the objects of interest are clearly visible. For each object several representations, i.e., levels of sparseness, are specified. The display of an individual object may incorporate different levels of sparseness. The goal is to emphasize important structures and to maximize the information content in the final image.

This paper also discusses several possible schemes for level of sparseness specification and different ways how object importance can be composited to determine the final appearance of a particular object.
\end{abstract}

Index Terms - view-dependent visualization, volume rendering, focus+context techniques, level-of-detail techniques, non-photorealistic techniques

\section{INTRODUCTION}

$\mathbf{T}$ HE importance of volumetric visualization in medical applications has been increasing significantly over the last years. In the future three dimensional visualization will be an essential tool for medical diagnosis. Due to the rapid development of high precision imaging modalities the amount of data keeps increasing. Therefore the need for emphasizing important structures raises.

Various medical applications exist where the size of the structures of interest is comparably small in relation to the overall data. Examples are examinations of tumors

The authors are with the Institute of Computer Graphics and Algorithms at the Vienna University of Technology, Austria. E-mail: \{viola,armin,meister\}@cg.tuwien.ac.at in the kidneys, lesions inside the liver and findings of lung nodules. In addition to that, performing diagnostic examinations is a complex task. Different features and properties of the anatomical tissues have to be taken into account. Especially not only the size and the shape of pathologies are of interest, but also their spatial position and vicinity to other anatomical structures. Hence, from a computer science point of view we are dealing with a focus and context task.

The investigation of liver lesions illustrates the medical requirements on the applied visualization method. Radiologists need to see the tumor from several directions in order to estimate the shape of the lesion. Computer aided diagnosis tools provide precise information about the size calculated from volumetric segmentation. The exact spatial position of arteries in close vicinity is very important in order to determine which liver segments to remove in a possible subsequent surgical treatment. Therefore it is necessary to visualize three different structures: the tumor, the vessel tree of the liver, and the liver parenchyma.

Visualizing these structures simultaneously - probably also including parts of the surrounding anatomy for orientation purposes - results in objects occluding each other. Known methods classify objects within the dataset independently from the viewpoint. This limits viewpoint positions and viewing angles to a range, where the important structures are not occluded by other objects. Using clipping geometries to reveal important interior structures eliminates less important objects also in those viewing situations where it would not be necessary. The application of different optical properties and rendering techniques (i.e., silhouette rendering) eases the problem only to a certain degree. From certain viewpoints several objects of less importance may overlap and largely obscure important features. Furthermore the fine-tuning of rendering parameters is a time consuming process not suitable for rapid clinical use.

These and similar visualization tasks can be resolved by importance-driven volume rendering (IDVR). The tumor and the vascular tree in close vicinity are the most important objects, the liver tissue and the surrounding 
anatomy (bones, aorta, skin) are of lower importance but still helpful for orientation purposes. Similar to anatomical drawings with IDVR the interesting structures are clearly visible from varying positions and different viewing angles. Occluding objects are rendered more sparsely or suppressed entirely.

The main contribution of this paper is importancedriven volume rendering as a model for automatic focus and context rendering. The proposed method overcomes the problem of occlusion within the volume, which happens when using any kind of view-independent classification. As opposed to previous approaches the optical properties of the proposed technique are not constant for an entire object. Depending on the viewing situation the level of sparseness varies dynamically. In order to visually emphasize features with the highest importance, objects between these features and the viewpoint are rendered very sparsely. Interesting objects are represented more densely to see most of the detail. Irrespective of the viewpoint there is always a clear view on the most important structures. If no occlusion occurs, even the less important objects can be rendered densely. This enables an automatic generation of images with maximal visual information.

In Figure 1 an anatomical illustration of the human abdomen [12] and a result of our technique is presented. In this case the internal structures are classified with a high importance value so that structures between the viewpoint and the important values are simply cut away automatically.
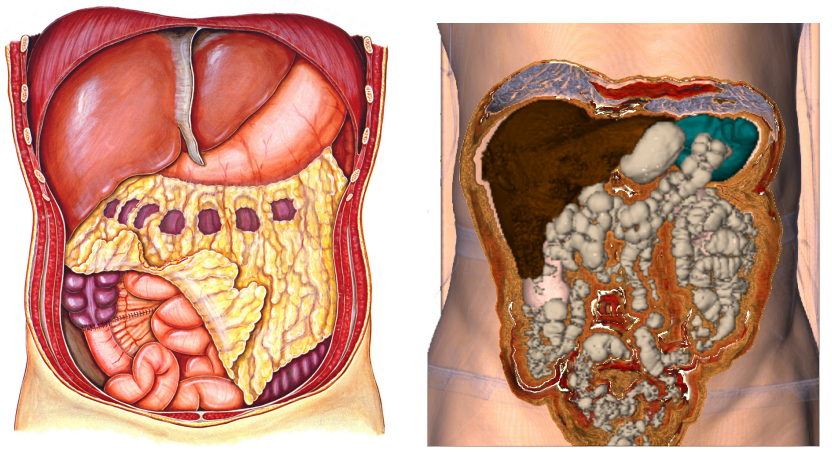

Fig. 1. Comparison between artistic medical illustration of the abdomen (left) and our method (right). Illustration image is courtesy to Howell MediGraphics.

The paper is organized as follows: Section II describes previous work related to importance-driven volume rendering. Section III explains the basic idea of the proposed model. Sections IV and V discuss the principal components of the model, i.e., importance compositing and levels of sparseness, and depict their impact on the resulting visualization. In Section VI we describe the test datasets and show various interesting rendering results applicable for medical and flow visualization. Finally we draw conclusions, summarize the paper in Section VII and propose future work in this field in Section VIII.

\section{RELATED WORK}

Scientific work related to our model can be divided into several categories. We will first discuss methods that use advanced and semi-automatic transfer function specification for feature enhancement. These classification methods are viewpoint-independent. Our work enables automatic focus+context visualization, where the information about the viewpoint is taken into account. We therefore point out some previous focus+context approaches. Afterwards we review various rendering techniques that are interesting for the level of sparseness specification. Finally we briefly mention other graphics research fields, where importance information plays a significant role.

Feature Classification: The most typical feature classification in volume visualization is the transfer function specification that maps sample density values to optical properties, i.e., color and opacity. Transfer functions with density as single input parameter are also denoted as onedimensional transfer functions. There has also been a lot of research on multi-dimensional transfer functions. The basic idea is to incorporate first and second derivatives of the density into the transfer function design [11], [17]. It is possible to assign optical properties based on gradient and curvature values, so for example object boundaries are classified differently from homogeneous regions. Taking into account first and second derivatives enables the semi-automatic generation of transfer functions [16]. An interesting approach was presented by Hauser and Mlejnek [9] for multidimensional 3D flow data. They use the Degree-of-Interest function that maps the user interest to optical properties.

Focus+Context Rendering: A lot of work has been done in the field of focus+context visualization. Viewpoint-dependent distortion of three-dimensional data [2] for example highlights data by dedicating more display space to it. Distortions are applied to abstract graphs in order to clearly see interesting graph nodes. An interesting idea is also to include the distance to focal point property into the volume rendering pipeline [30]. This allows to change the optical properties according to the distance to the focal point. Using this technique several expressive focus+context effects can be achieved. Gaze-directed volume rendering takes the observer's viewing direction into account to increase the rendering performance [18]. The volume dataset is rendered in 
different resolutions. According to the viewing direction the focal region is represented in full resolution, while the other parts are rendered in lower resolution.

Sparse Representation: The graphics community has been inspired by artists to reduce a visual representation just to show features of interest. This is in contrast to traditional photorealistic approaches. Nonphotorealistic methods show features in a sparse way exploiting human imagination. The display of contours is a popular method to represent context information in volume visualization [3], [22]. Outlines are often sufficient to roughly understand the shape and can be combined with other rendering techniques like direct volume rendering or maximum intensity projection [10]. To make a contour representation more dense suggestive contours can be introduced [5]. Additionally to real contours also contours from virtual viewpoints close to the current view are rendered. Also pen-and-ink techniques convey good shape information. Pen-and-ink styles in combination with traditional volume rendering have already been applied for focus+context rendering in volume visualization [27]. This is up to a certain degree similar to combining curvature-directed strokes with isosurface rendering [13]. This approach was proposed for rendering structures that are completely enclosed by other objects. The interior structures are rendered fully opaque, while the enclosing objects are represented by a set of curvature-directed lines. The visibility of interior structures can also be modified by dynamic changes in transparency of the outer shape. Dynamic transparency is also used in user interface design [8].

Cut-Away Views: Cut-away illustrations are another way to represent nested objects. The popularity of this technique is demonstrated by the fact that it can be found in almost all books with technical or medical illustrations. In volume visualization this technique is also known as volume cutting [21]. Straka et al. [24] are applying a cut-away technique for CT-Angiography. Diepstraten et al. [6] describe how various cut-away techniques can be achieved automatically. Streamarrows were presented by Löffelmann et al. [19] for visualizing complex dynamical systems. They use arrows as a basic element for cutting away part of the stream surface. This allows to see through the surface and perceive other surfaces or structures behind.

Importance-Driven Rendering: Importance as a rendering parameter has already been investigated in other graphics fields. In global illumination the term importance is used quite often. Importance sampling for example is a popular method to accelerate convergence of Monte-Carlo integration by faster reducing the variance. The basic idea distributes sample points non- uniformly [14], [26]. The idea of importance sampling has been recently researched also for volume rendering [4], [7]. For radiosity techniques importance can also encode the potential visibility of surfaces [23] or the prominence of a patch as a light source. Importance is also proposed as an additional user-defined parameter for halftoning [25]. All these methods employ importance in different contexts and meanings. But all of them use importance as an additional dimension to improve the behavior of traditional approaches. In the following we discuss how importance is applicable also for volume rendering. Importance-driven volume rendering has already been presented in our previous work [29].

\section{IMPORTANCE-DRIVEN VOLUme RENDERING}

In volume visualization we are often dealing with the problem that interesting structures are partly or completely occluded by surrounding tissue. This is hard to resolve by traditional view-independent approaches, such as transfer function specification. We propose a viewpoint-dependent model that removes unwanted occlusions automatically and maximizes the information content in the final image.

Traditionally features within the volume dataset are classified by optical properties like color and opacity. We additionally assign another dimension to features, which describes their importance. Importance encodes which features are the most interesting ones and have the highest priority to be clearly visible. Each feature is therefore weighted by a positive scalar value called object importance. During the rendering stage, the model evaluates the visibility of each feature (also denoted as object) according to its importance. If less important objects are occluding features that are more interesting, the less important ones are rendered more sparsely, e.g., more transparently. If the same object does not cause any unwanted occlusions in other regions of the image, it is rendered more densely, e.g., opaque, in order to see its features more clearly. This allows to see all interesting structures irrespective if they are occluded or not, and the less important parts are still visible as much as possible. Instead of using constant optical characteristics, which are independent from the viewpoint, we use several levels of sparseness for each object. We do not assign a single optical characteristic, but several characteristics with smooth transitions inbetween. These multiple levels of sparseness allow the object to continuously change its visual appearance from a very dense representation to a very sparse one. Which level of sparseness will be chosen is dependent on the importance of the particular object and the importance of hidden objects behind. The level of sparseness thus may continuously vary within a 


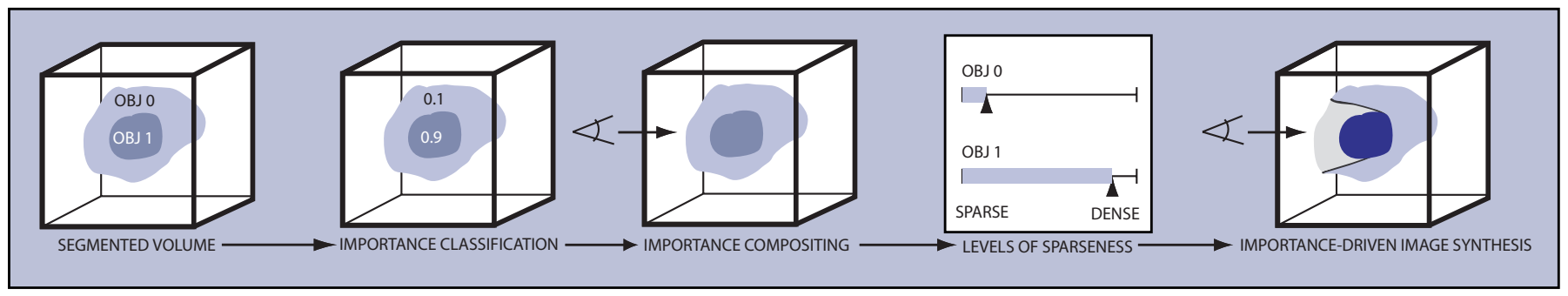

Fig. 2. Stages of the pipeline for importance-driven volume rendering: Volumetric features are assigned by importance values. The volume is traversed in the importance compositing stage to estimate levels of sparseness. These are used to enhance or suppress particular parts of the volume. The resulting images emphasize important features.

single object. Also depending on the viewpoint the same part of an object may be represented with different levels of sparseness. To determine the sparseness level for each object or parts thereof the rendering pipeline requires an additional step, which we call importance compositing. This step evaluates the occlusion, takes the importance factor of each object into account and assigns to each object particular levels of sparseness. The final synthesis results in images with maximal visual information with respect to the predefined object importance. For the sake of clarity the controlling components of importancedriven volume rendering are now briefly summarized:

Object importance describes the visibility priority of each feature within the volume dataset. It is a positive scalar value, which is constant for the whole object. The feature (or object) can be determined in many different ways, which also depend on the type of the data. In medical visualization features are often classified as particular organs. Such objects are defined by a segmentation process. Another way of feature classification can be through a spatial relationship within the volume or eventually through the location with respect to another feature. In such way it is for example possible to classify the vortex core of a hurricane. In case of multidimensional volumetric data features can be defined by specifying interesting ranges of values for each data dimension. There are many other ways to determine features. A detailed treatment of feature definition is outside the scope of this paper.

Levels of sparseness are various different representations of a particular feature from the most dense to the most sparse one. Sparseness is loosly defined in terms of how much the display of an object takes up screen estate. For an iso-surface a point-cloud or a wire-frame display is a very sparse representation. The display of filled opaque polygons is a very dense representation of the same iso-surface. In case of volumetric data each sample within the volume is classified to have a color and opacity. In direct volume rendering (DVR) these values are composited together to synthesize the final image. The sparseness can in this case modulate the opacity of object samples so that the most interesting samples are opaque while other samples are more transparent. Varying transparency is a simple and straightforward example of levels of sparseness. More elaborate schemes are discussed in Section V.

Importance Compositing is an additional rendering step that assigns to each part of an object a specific level of sparseness. A very simple importance compositing scheme is maximum importance projection (MImP). For each image area only the object with highest importance is visible. The remaining objects are shown with the highest level of sparseness, i.e., fully transparent. This results in cut-away views of important structures. As another example, we can perform importance compositing in a similar way as compositing of color and opacity in the traditional DVR approach. Instead of compositing optical properties, the object importance values are accumulated. We will discuss different compositing modes in Section IV.

The interrelationship between the above mentioned components is depicted in Figure 2. The importance compositing is done similar to the DVR approach. For each ray the compositing evaluates object occlusions and assigns the corresponding level of sparseness to each object. Object importance is presented in the sense that it is mapped to object visibility in the result image. This causes different rendering settings for the context object $O B J O$ in the area of the image which is covered by the focus object $O B J 1$ (see Figure 2). The difference between traditional volume rendering and importancedriven volume rendering is clearly visible in Figure 3. The goal is to emphasize the inner organ as focus object. In the traditional approach it is necessary to reduce the opacity of occluding objects globally. Importance-driven rendering assigns a higher sparseness factor only to the area where occlusion occurs. 


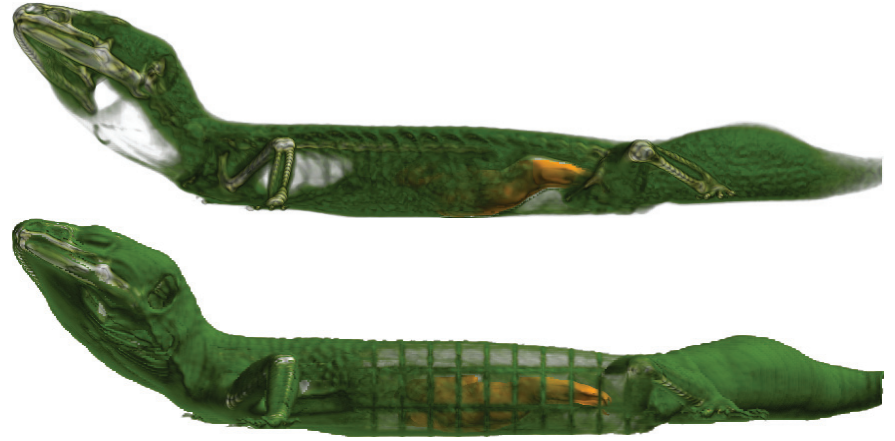

Fig. 3. Comparison between traditional volume rendering (top) and importance-driven volume rendering (bottom). The orange inner organ is the focus object

\section{IMPORTANCE COMPOSITING}

Importance compositing is an additional pass added to the traditional volume-rendering pipeline. It determines the level of sparseness for each object or a part thereof in order to preserve important features. There are many possibilities conceivable how to perform importance compositing. In the following we will discuss three methods of importance compositing which are inspired by compositing optical properties through ray casting of volume data.

\section{A. Maximum Importance Projection}

Maximum intensity projection (MIP) [20] is a simple and fast volume rendering approach. It is applicable for sparse data where important information has high intensity values like contrast-media enhanced blood vessels. With MIP compositing reduces to selecting the highest intensity value along a ray. Intensities are encoded as gray values to produce the final image.

Analogous to MIP we propose maximum importance projection $(M \operatorname{Im} P)$. For each ray the object with highest importance along the ray is determined. This object is displayed densely. All the remaining objects along the ray are displayed with the highest level of sparseness, i.e., fully transparent. With $M \operatorname{ImP}$ structures are either rendered using the most dense representation or they are not rendered at all.

With MIP the spatial arrangement of structures is not readily apparent. MImP has a similar problem which we alleviate as follows. The image area, where the most important object is projected onto is denoted as object footprint. With MImP the footprint is exactly the image region where only the object is visible. One can consider MImP as a cut-away view, where the space in front of the most important object is simply clipped. The clipping object is a translational sweep with the footprint as cross section (general cylinder). One can now modify this cylinder to obtain a clipping frustum. This is achieved by scaling up the footprint during the translational sweep towards the viewer. This produces a countersink clipping geometry. Figure 4 illustrates the difference between the above described cylindrical and a conical MImP in 2D. The conical MImP is easily realized during rendering by changing the starting point during ray traversal for those rays intersecting the side faces of the clipping frustum. Figure 5 shows images to compare both approaches.

The countersink geometry, respectively the ray starting points are computed from the footprint of the focus object. The footprint contains depth information of the focus objects' last hit for each ray along the viewing direction. This information is used for performing the cutout. For cylindrical MImP we simply skip ray samples that belong to the context object, until the focus objects' last hit depth is reached. For the conical MImP we need to enlarge the footprint to build the conical shape. This is possible to realize using image processing operators on the depth image, where the intensity encodes the depth of the entry point. The depth-footprint is processed by a 2D chamfer distance transform [1]. The highest depth value of the footprint is calculated. The depth buffer of ray starting points that contribute to the countersink $e_{i}$ are calculated from the highest depth value $e_{\max }$, the slope $s_{c}$ of the countersink and the distance value $d_{i}$ at pixel $i$ as shown in Equation 1 .

$$
e_{i}=e_{\max }-d_{i} * s_{c}
$$

To correctly simulate the cut-out it is necessary to change the gradient vector at the entry points that contribute to the countersink effect. Two components of the gradient are estimated from the gradient information of the $2 \mathrm{D}$ distance field. The $z$ component is constant, i.e, the slope of the countersink frustum.

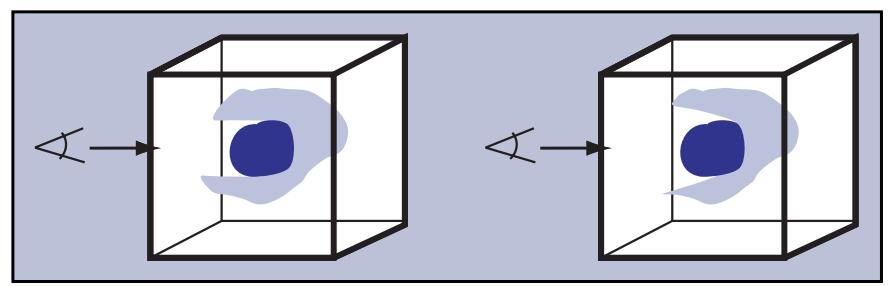

Fig. 4. Maximum Importance Projection. Illustration of cylindrical (left) and conical countersink (right).

\section{B. Average Importance Compositing}

The second approach of importance compositing takes into account all the objects along a ray. The influence of an individual object is hereby independent from the number of ray samples within the object. An object $O$ 

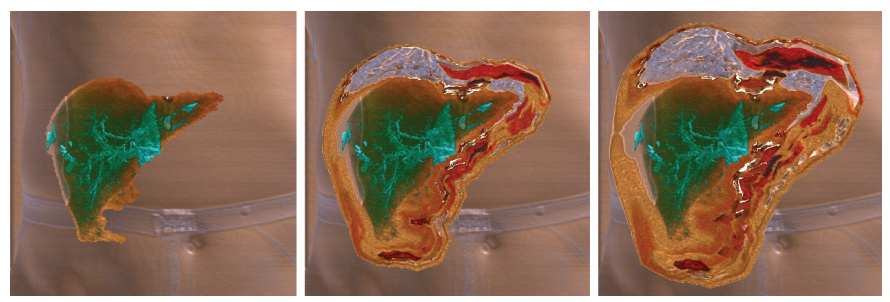

Fig. 5. Maximum importance projection (MImP). Cylindrical MImP (left) does not clearly show the spatial relationships between focus and context objects, i.e., the focus object appears in front of the context object. Conical MImP corrects this visual artifact. Different slope factors define the countersink geometry (center and right).

has an importance value $I_{o}$. Ray $r$ is intersected by $n_{r}$ objects. The level of sparseness $S_{o}$ of a particular object $o$ at ray $r$ is equal to the fraction of its own importance and the sum of the importance of all the intersected objects:

$$
S_{o}=\frac{I_{o}}{\sum_{i=1}^{n_{r}} I_{i}}
$$

Average importance compositing (AImC) does not completely remove the less important objects as with MImP. The sparseness factors are estimated according to the given importance. This allows a very sparse representation of the occluding object to see a rough structure of the shape and to clearly see the important object behind it. The importance compositing stage computes the levels of sparseness of all objects for every pixel in the final image. Levels of sparseness are computed using the object footprints. At each pixel position we perform a lookup to each object footprint. Object importance values of all objects that have the footprint at current pixel position are summed together. The sparseness factor for each of these objects is estimated through division of their object importance by the evaluated sum.

The final image synthesis using $\mathrm{AImC}$ is an extension to traditional DVR. During the ray traversal at each sample location the level of sparseness additionally modulates the visibility of the sample. However it generates images where the spatial arrangement of structures is not readily apparent similarly to cylindrical MImP. In order to correct the spatial arrangement we propose two methods to perform final importance-driven image synthesis using AImC, i.e., image-space and object-space approach.

Image-Space AImC: The original object footprints exhibits sharp transitions in levels of sparseness, which cause confusing spatial arrangement. To correct the spatial arrangement we generate smooth transitions between different levels of sparseness. Before the levels of sparseness of each object are computed we apply image processing operators, i.e., dilation and averaging to every footprint. The levels of sparseness estimation is analogue to Equation 2. For each pixel we compute the sum of all contributing objects, i.e., where the footprint value is not equal to zero. The object importance is in this case always multiplied by the footprint value in the range of $[0,1]$. Footprint values below one are part of the transition area between different levels of sparseness.

The image-space approach does not evaluate whether the sample of a suppressed context object is in front or behind the important object. The level of sparseness is constant for all samples of one object along particular ray. This means that also part of context object behind the focus object is suppressed.

Object-space AImC: To avoid suppression of context behind the focus object we propose object space approach. Using this approach only those samples of the context object are suppressed that are in front of the focus. The level of sparseness is in this case not constant for one object along a particular ray. The difference between image-space and object space approach is illustrated in Figure 6. The image synthesis of the

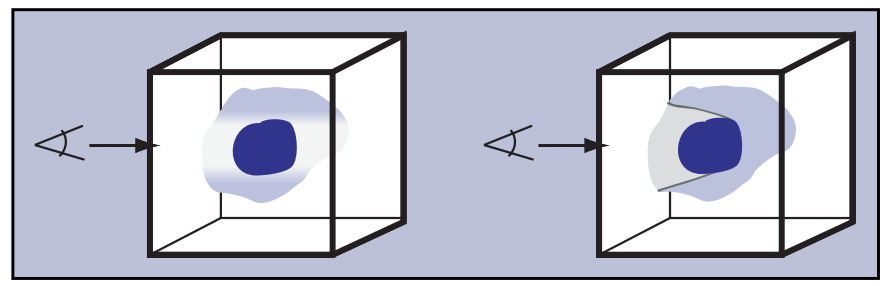

Fig. 6. Average importance compositing (AImC). The illustration depicts the difference between the image-space (left) and object space approach (right). While image-space AImC suppresses all context samples along the ray, the object space approach suppresses only the part of context object that occludes the focus object.

object space approach is analogue to the conical MImP. The countersink geometry in case of conical MImP is used for estimation of starting position of the ray in order to perform the cut-out. In object-space AImC the countersink geometry defines the region of different level of sparseness. The starting position of the ray is not changed. During the ray traversal of final image sythesis, each sample location is evaluated whether it belongs to the countersink area or not. Outside the countersink area is the context represented using more dense representation and inside more sparse form is chosen.

Results of each approach are shown in Figure 7. The images show the same dataset under different viewing angles. The top row shows the image-space approach and the bottom the object-space.

AImC approach is preserving the thickness of the 


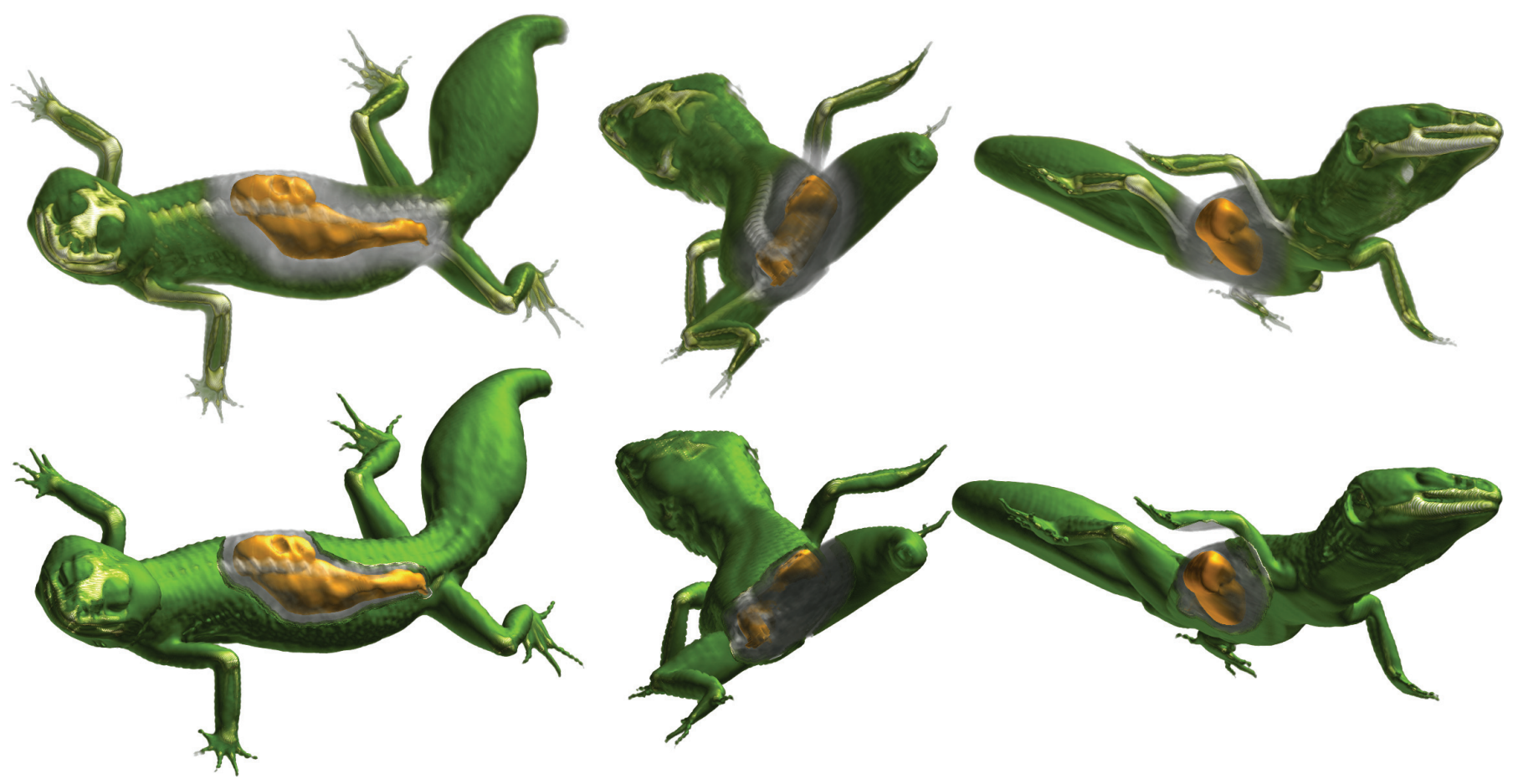

Fig. 7. Average importance compositing (AImC) is shown under different viewpoint settings in combination with modulating optical properties. Upper row shows image based AImC. The bottom row shows the object space approach with the same viewpoint settings.

occluding part of the context object. This leads to different visibility of the focus object under different viewing conditions. If the occluding context area is too thick, the focus object is not visible enough. In order to see the focus object properly the level of sparseness function has to be changed for the context object or the importance of the focus object has to be increased. Another approach how to overcome the problem of varying thickness sof the occluding object is described in the following subsection.

\section{Visibility Preserving Importance Compositing}

Visibility preserving importance compositing (VPImC) guarantees constant visibility of the focus object. Independently from the thickness of the context volume part, which is in front of the focus, there is a constant fraction of the visual information for the focus object. For example under some viewing angle is the context object thin and samples can be represented more dense. Under different viewing angle the context object can be more thick in front of the focus object. Therefore samples that belong to this area should be more transparent. This is illustrated in Figure 8.

The values for levels of sparseness using VPImC are estimated in the same way as in case of AImC (Equation 2). In AImC the levels of sparseness are selected during the rendering ray-traversal for each sample. With VPImC we select an appropriate level of sparseness after the rendering stage. This obviously cannot work for every level of sparseness scheme. The level of sparseness is applied as follows: The desired level of sparseness for the context object in front of the focus object is $S_{o}$. In VPImC the goal is to adjust the average accumulated opacity of the occluding region to be equal to the value $S_{o}$. Therefore the context part in front of focus is rendered separately. All ray opacities of the occluding part are summed together to compute the average perray opacity. To keep the constant visibility of the focus object the average per-ray opacity has to be equal to $S_{o}$. Therefore for each ray the original accumulated opacity value is corrected. This is expressed by the following Equation 3:

$$
\alpha_{\text {accum_new }}(x)=\alpha_{\text {accum }}(x) \frac{S_{o}}{\alpha_{\text {avg }}}
$$

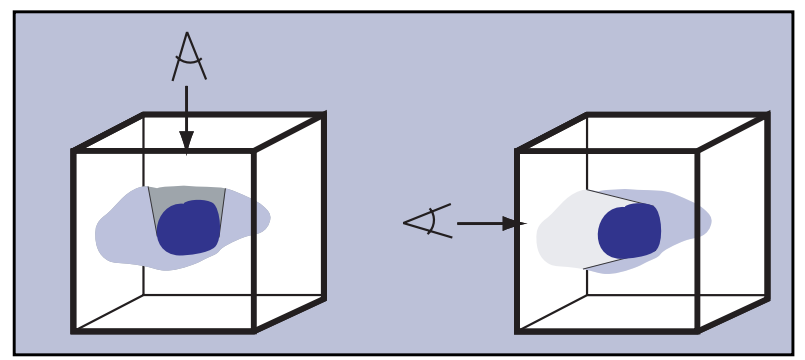

Fig. 8. Visibility preserving importance compositing. The suppression of the context varies according to the thickness of the context. 

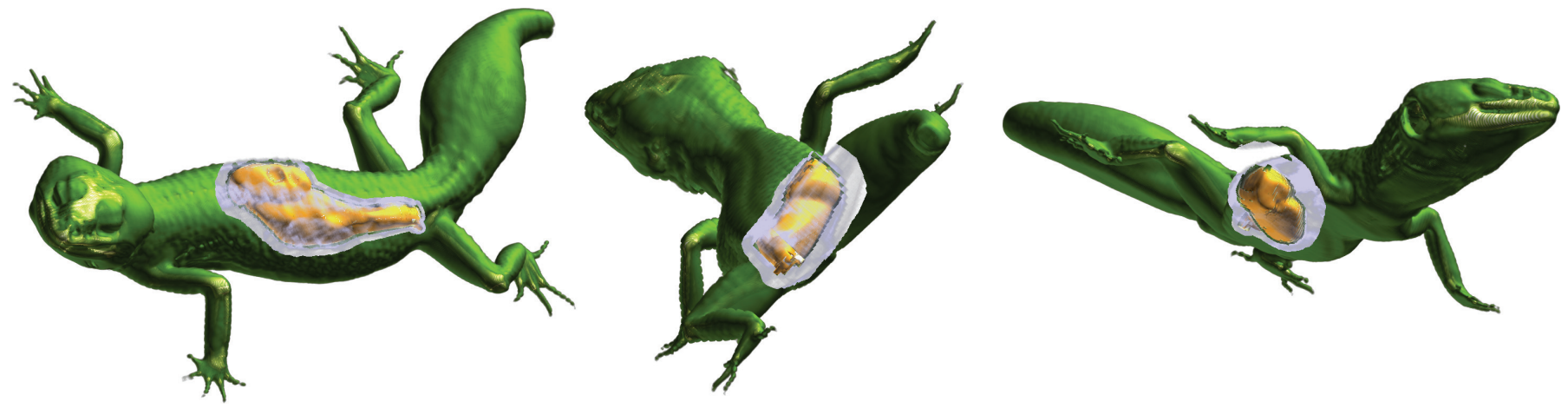

Fig. 9. Visibility preserving importance compositing (VPImC). The dataset is shown under different viewpoint settings with constant visibility of the focus object which is independent from the thickness of the occluding context part.

where $\alpha_{\text {accum_new }}$ is the modified accumulated opacity of the suppressed context part, $\alpha_{\text {accum }}$ the original density value, $S_{o}$ the level of sparseness value of the context object and $\alpha_{\text {avg }}$ is the average accumulated opacity value of the suppressed context part.

The separate rendering of the occluding part is done using two level volume rendering [10]. Every object or its part (as in case of the context object) are rendered separately in a local compositing step. Then the visibility correction is done for the occluding part. Finally a combination of both context parts takes place in the global compositing step.

Figure 9 shows the results of the compositing under different viewpoint settings. It offers a clear comparison to the importance compositing technique shown in Figure 7 (described in Section IV-B).

\section{LEVELS OF SPARSENESS}

The importance compositing determines for each object respectively parts thereof the visibility on the rendered image by determining the level of sparseness. This section describes three schemes how to achieve transitions of object visibility.

These schemes are compared in Figure 10, which shows the Leopard Gecko dataset, where the interior part was pre-segmented. The series of images illustrates how the context area in front of the focus object smoothly varies from a dense to a sparse representation.

\section{A. Color and Opacity Modulation}

A direct control of optical properties is the first approach to modify the visual prominence of a particular object. With increasing sparseness the object becomes more transparent in order to show the more important underlying data. This approach is widely used in transfer function specification.
Interesting results can be achieved by controlling color saturation with the level of sparseness factor. Color is a very important visual cue for visualization in general. Highly saturated colors are attracting the observer's attention more than colors close to gray. The level of sparseness can therefore be expressed also in the saturation of the color. Changing only the saturation, however, does not increase the visibility of occluded objects. It is necessary to change the color and opacity values at the same time. Different visual appearances within the same object can cause misinterpretations. Therefore smooth transitions between different levels of sparseness have to be applied. A smooth modulation of the optical properties is shown in Figure 10 (top).

\section{B. Screen-Door Transparency}

Screen-door transparency is a well-known strategy to simulate transparency. The semi-transparent view of an object behind a screen-door is simulated as follows: A screen-door consists of a mesh and holes inbetween. The mesh fully occludes the object behind, whereas it is fully or partly visible through the holes. From a certain distance holes and mesh blend together to produce a semi-transparent impression. We use an analogue idea to define levels of sparseness. The volumetric dataset consists of voxels. The level of sparseness determines which voxels should be rendered and which not. The distribution of visible voxels is uniform and is forming a wireframe-like structure. The impact of increasing sparseness is shown in Figure 10 (middle).

\section{Volume Thinning}

Volume Thinning proceeds as follows: Voxels of an object are sorted according to two sorting keys. The first sorting key is gradient magnitude, the second sorting key is curvature magnitude of the iso-surface through the voxel. Reducing the sparseness factor according to 

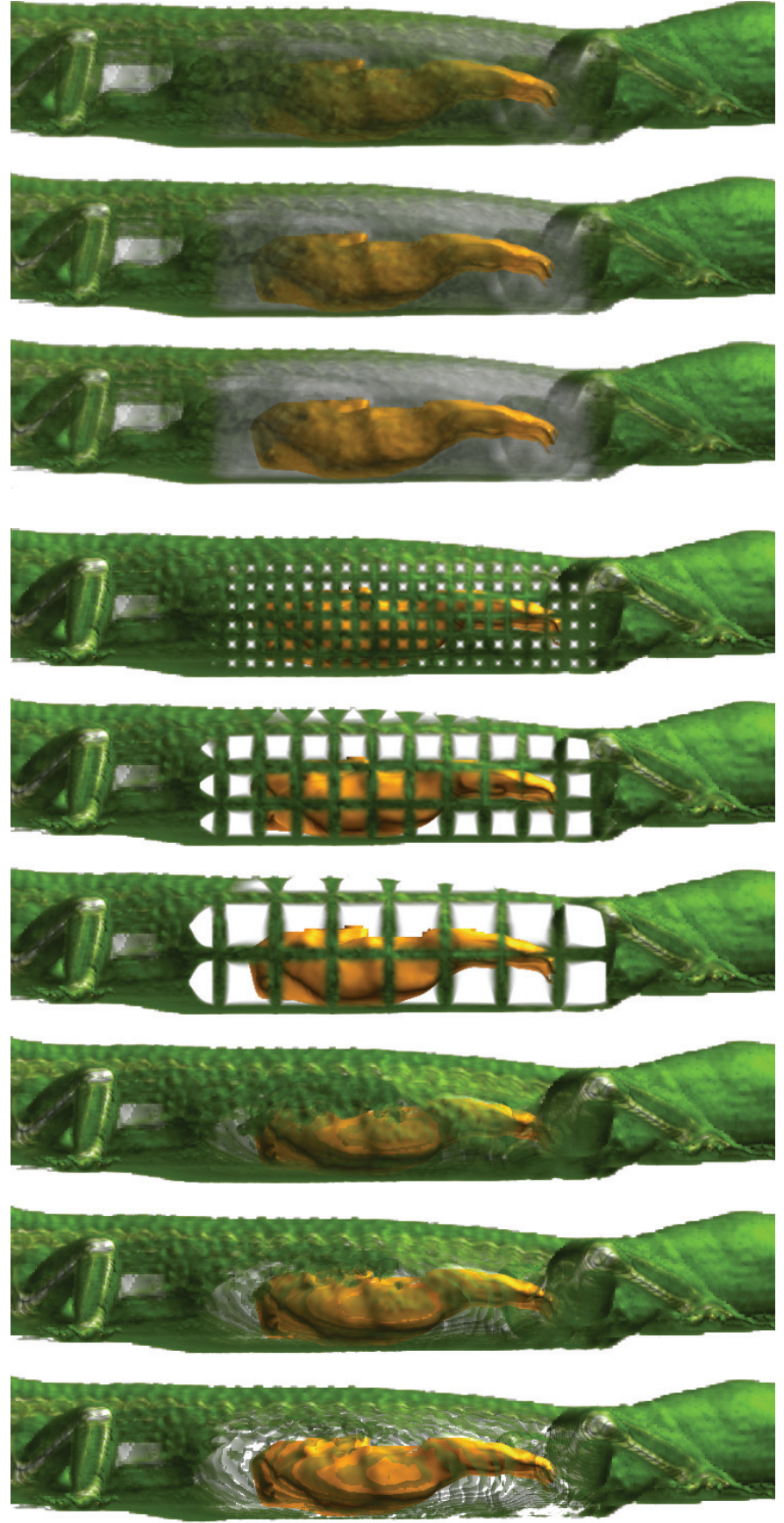

Fig. 10. Changing levels of sparseness. Top three rows: opacity modulation and color saturation modulation. Middle three rows: screen-door transparency. Bottom three rows: volume thinning. Images display levels of sparseness with factors $0.75,0.5$, and 0.25 .

gradient magnitude has the effect that the volume is continuously reduced to fewer and fewer strong iso-surfaces. As soon as only few iso-surfaces remain the reduction proceeds according to curvature magnitude. This has the effect that the iso-surfaces gradually dissolve and in the end (most sparse representation) only few high curvature areas remain. Figure 10 (bottom) illustrates visibility reduction through volume thinning.

\section{Sparse and Dense Rendering Styles}

Previous levels of sparseness techniques describe how to enhance/suppress the visual representation of a particular object. The sparseness function smoothly varies from the most dense to the most sparse representation.

Another way how to define levels of sparseness function can be assignment of different rendering techniques as different levels of sparseness. For example the dense representation can be represented by direct volume rendering and the sparse one by non-photorealistic contour rendering. In case of object representations the combination is done via compositing. The way how to combine different rendering techniques is achieved by the two level volume rendering concept [10]. The difference of levels of sparseness function based on object representations and rendering style is shown in Figure 11.

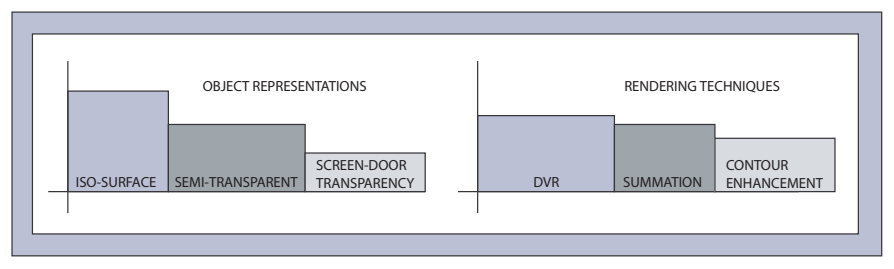

Fig. 11. Two kinds of levels of sparseness function: function based on object representations (left) and levels of sparseness function using different rendering techniques (right).

Two level volume rendering is a technique to combine different volume rendering techniques together. Wellknown rendering techniques are direct volume rendering (DVR), MIP, summation (similar to X-Ray imaging), or non-photorealistic rendering with contour enhancement (NPRCE) [3], [22]. 2IVR allows to render each object within a volume with a different technique and composite the optical properties in a local compositing step. Each ray is partitioned by the intersecting objects into sub-rays. Local compositing is done for each subray according to the rendering technique chosen for the respective object. The result of an entire ray is achieved in a global compositing step which combines the results of the individual sub-rays.

The results of using different rendering techniques in levels of sparseness function are shown in Figure 12. The context information is rendered using standard DVR where no occlusion occurs. In case of occlusion of the inner structure a different sparse rendering technique is applied. Images show the application of summation, contour enhancement, and maximum intensity projection for the local compositing. Global compositing is done by using DVR. 


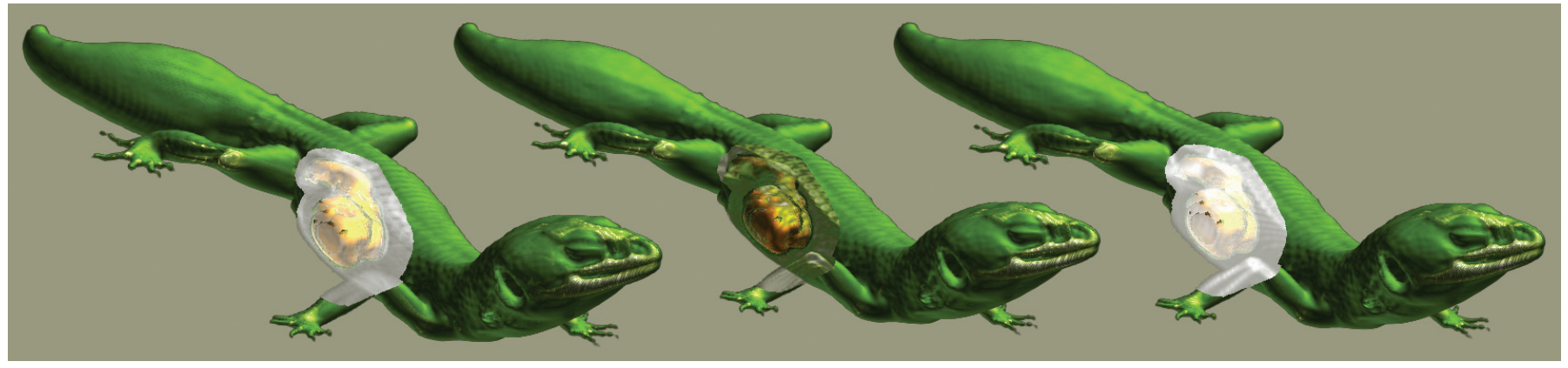

Fig. 12. Sparse and dense rendering styles: The context object part that occludes the important information is rendered using summation, non-photorealistic contour enhancement rendering technique, maximum intensity projection.

\section{RESULTS}

We show results of our method on three datasets. The Leopard Gecko dataset is of resolution $512 \times 512 \times 87$. The Monster Study dataset has been downsampled to the half of its full resolution, i.e., $256 \times 256 \times 610$. Both datasets are using pre-segmented objects. The Monster Study has all important organs pre-segmented. Therefore it is optimal for automatic generation of medical illustrations. The third dataset is a time-varying simulation of the hurricane Isabel. It is a three-dimensional flow dataset with multiple simulated properties including cloud moisture, precipation, pressure, and temperature. This dataset is not pre-segmented, only the position of the hurricane eye in object space is predefined. The simulation consists of 48 time steps.

Figure 13 shows the conical MImP of multiple abdomen organs from different viewpoints. The liver, milt, kidneys, and intestine have the same importance value. The tumor (in yellow) located between kidney and liver, has the highest importance value. The rest of the body is of lower importance than any of mentioned objects. The footprints of the abdomen organs are the basis for the generation of the countersink geometry. The organs of the abdomen have the same importance and therefore they do not cut away each other. The highest importance value is assigned to the tumor and therefore everything in front of the tumor is cut-away.

Another example of a conical MImP is shown in Figure 14. It shows 6 out of 48 time steps of the hurricane Isabel data. In this case a different way of feature classification was chosen. The important feature is the position of the eye of the hurricane. At the eye position a proxy cylinder is placed and everything inside the cylinder has higher importance than the rest of the data. The cylinder footprint is the basis for the countersink geometry.

This example also shows how to combine multiple scalar volumes using importance-driven volume ren- dering. The focus object is defined as the group of voxels inside the cylinder around the hurricane eye. Inside the cylinder the total precipation mixing ratio is visualized. Thanks to the cut-away view it is possible to have a clear view at this property close to the eye of the hurricane. Outside the cylinder is the context area where the total cloud moisture is visualized. This timedependent dataset also shows that the important feature can change its position during the time. So in this case not only the viewpoint can change, but also the cylinder position. Importance-driven volume rendering guarantees to see the important feature irrespective of viewpoint and feature position.

The performance of the current implementation is not interactive. The goal of the implementation was to do a proof of concept rather than performance optimizations. The model was integrated as a plugin into the J-Vision [15] medical workstation.

To fully appreciate the strengths of importancedriven volume rendering viewpoint changes or dynamic scenes are essential. This is best illustrated with animation sequences, which are available at http://www.cg.tuwien.ac.at/research/vis/ adapt/2004_idvr/.

\section{SUMMARY AND CONCLUSIONS}

In this paper we have proposed importance-driven volume rendering as a view-dependent or feature dependent model for automatic focus+context volume visualization. A new factor to the traditional volume rendering pipeline is introduced, i.e., the importance dimension. According to the importance and viewpoint settings each object is rendered in order to maximize the visual information. This method allows to see structures within the volume as dense as possible. A sparse representation is chosen only if other, more important structures are occluded.

Importance compositing defines how the occluding context information should be visualized. It can be simply cut away (MImP), or displayed using sparse representations. This approach can preserve either the thickness of the context object or the constant visibility of the focus object. 

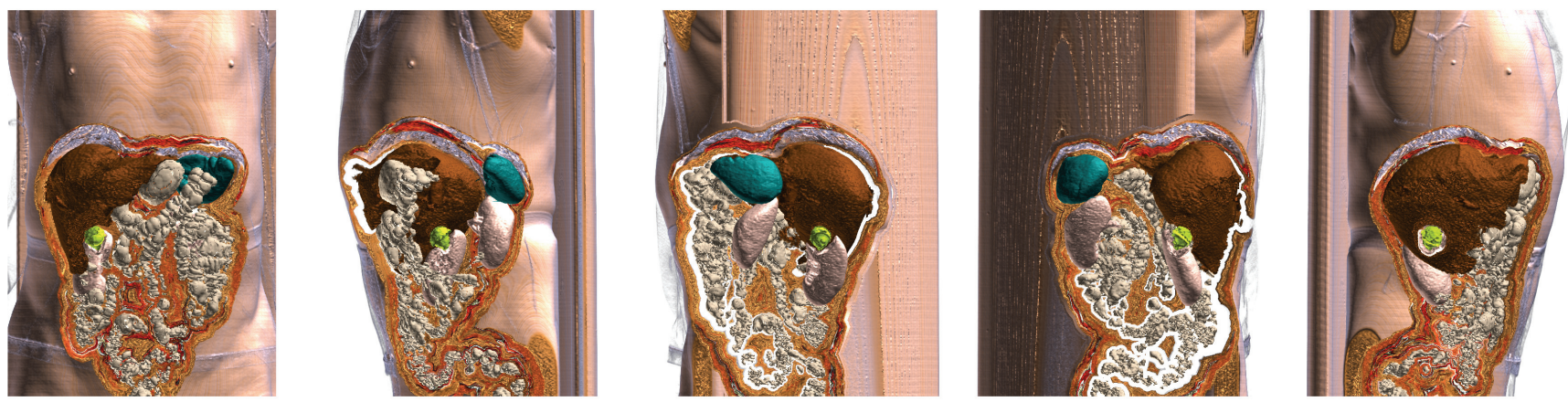

Fig. 13. The Monster Study dataset rendered using conical MImP. The highest importance is assigned to the tumor object (yellow). The organs of the abdomen are assigned a lower importance. The rest of the dataset has the lowest importance.
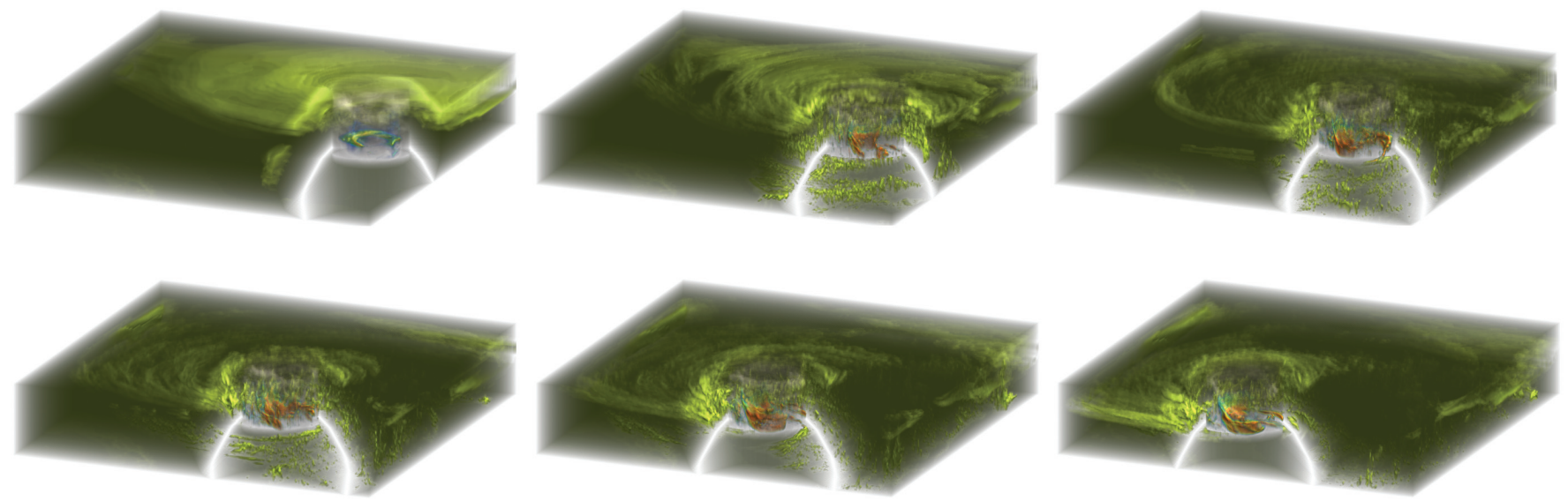

Fig. 14. Visualizing the simulation data of hurricane Isabel. Two different properties are visualized: total cloud and total precipitation mixing ratios. The interesting feature is the precipitation close to the eye of the hurricane. The context feature is the cloud mixing ratio. Images are showing 6 out of 48 time steps from left to right, top to bottom.

We have discussed four schemes for levels of sparseness. Levels of sparseness control the optical properties or the amount of visible elements of the volume. Smooth opacity changes work well in combination with desaturation. The amount of visible volume elements can be distributed uniformly over the whole volume, or the first- and second-order derivatives can be used for visibility distribution.

The levels of spaseness function is used for transitions of data representation from most dense to most sparse. The second approach is to define levels of sparseness with different rendering techniques from the most dense to the most sparse one.

\section{FUTURE WORK}

The paper opens a lot of opportunities for possible research areas. We assume that the objects within the volume data are pre-defined by segmentation or other feature classification methods and the importance is assigned manually by the user. An open issue is, how to do the feature selection and importance assignment automatically. Various automatic feature detection approaches can be integrated into the model to select the important features without additional user interaction.
The paper has presented various levels of sparseness schemes. The continuous transition from dense to sparse representations for volumetric data is a wide area of research. In polygonal rendering levels of sparseness are widely used. The most sparse representation is a set of points, another representation is a wireframe display, and the most dense display is a surface representation. Volume graphics does not yet have such a variety, which shows the need for research in this area.

The third factor of importance-driven volume rendering is importance compositing. The paper presents simple compositing schemes derived from ray-casting approaches. The next step are compositing schemes that incorporate first- and second order derivatives to preserve object boundaries. The parts with high first derivatives values can be then considered as more important and a dense representation is chosen.

The conical MImP and other object-space importance compositing approaches are using the cut-out illustration technique to improve perception of the spatial relationships. More elaborate approaches for intelligent automatic cut-out generations are to be researched more intensively. In cut-away views sometimes the borderline of the cutted regions is emphasized 
(e.g., through thick lines, or zig-zag lines). Automatically emphasizing these transition zones is also an open problem.

Each viewpoint brings out only a fraction of the entire information encoded in the data set. How to estimate viewpoint entropy and how to automatically determine optimal viewpoints [28] is another, not yet researched, area for volume data.

\section{ACKNOWLEDGMEnTs}

The work presented in this publication has been funded by the ADAPT project (FFF-804544). ADAPT is supported by Tiani Medgraph, Vienna (http://www.tiani.com), and the Forschungsförderungsfonds für die gewerbliche Wirtschaft, Austria. See http://www.cg.tuwien.ac. at/research/vis/adapt for further information on this project.

The Monster Study dataset is courtesy of Tiani Medgraph and the Leopard Gecko is courtesy of University of Veterinary Medicine Vienna. The hurricane Isabel data is courtesy of the National Center for Atmospheric Research in the United States. The medical illustration of the human abdomen is courtesy of Howell MediGraphics [12].

\section{REFERENCES}

[1] G. Borgefors. Distance transformations in digital images. Computer Vision, Graphics, and Image Processing, 34(3):344371, 1986.

[2] M. S. T. Carpendale, D. J. Cowperthwaite, and F. D. Fracchia. Distortion viewing techniques for 3-dimensional data. In Proceedings of IEEE Symposium on Information Visualization '96, pages 46-53, 1996.

[3] B. Csébfalvi, L. Mroz, H. Hauser, A. König, and M. E. Gröller. Fast visualization of object contours by non-photorealistic volume rendering. In Procceedings of EUROGRAPHICS '01, pages 452-460, 2001.

[4] B. Csébfalvi and L. Szirmay-Kalos. Monte carlo volume rendering. In Proceedings of IEEE Visualization '03, pages 449-456, 2003.

[5] D. DeCarlo, A. Finkelstein, and S. Rusinkiewicz A. Santella. Suggestive contours for conveying shape. In Proceedings of ACM SIGGRAPH '03, pages 848-855, 2003.

[6] J. Diepstraten, D. Weiskopf, and T. Ertl. Interactive cutaway illustrations. In Procceedings of EUROGRAPHICS '03, pages 523-532, 2003.

[7] S. Grimm, S. Bruckner, A. Kanitsar, and E. Gröller. VOlume doTS as a point-based representation of volumetric data. In Proceedings of EUROGRAPHICS '04, pages 661-668, 2004.

[8] C. Gutwin, J. Dyck, and Ch. Fedak. The effects of dynamic transparency on targeting performance. In Proceedings of Graphics Interface '03, pages 105-112, 2003.

[9] H. Hauser and M. Mlejnek. Interactive volume visualization of complex flow semantics. In Proceedings of $V M V$ '03, pages 191-198, 2003.

[10] H. Hauser, L. Mroz, G. I. Bischi, and M. E. Gröller. Twolevel volume rendering. IEEE Transactions on Visualization and Computer Graphics, 7(3):242-252, 2001.

[11] J. Hladůvka, A. König, and E. Gröller. Curvature-based transfer functions for direct volume rendering. In Proceedings of SCCG '00, pages 58-65, 2000.

[12] Howell MediGraphics web site, http://www.medigraphics.com/, 2004.
[13] V. Interrante, H. Fuchs, and S. Pizer. Illustrating transparent surfaces with curvature-directed strokes. In Proceedings of IEEE Visualization '96, pages 211-218, 1996.

[14] H. Wann Jensen. Importance driven path tracing using the photon map. In Proceedings of Rendering Techniques '95, pages 326-335, 1995.

[15] J-Vision medical workstation, Tiani MedGraph web site, http://www.tiani.com/, 2004.

[16] G. Kindlmann and J. Durkin. Semi-automatic generation of transfer functions for direct volume rendering. In Proceedings of IEEE Symposium on Volume Visualization '98, pages 79-86, 1998.

[17] J. Kniss, G. Kindlmann, and Ch. Hansen. Interactive volume rendering using multi-dimensional transfer functions and direct manipulation widgets. In Proceedings of IEEE Visualization '01, pages 255-262, 2001.

[18] M. Levoy and R. Whitaker. Gaze-directed volume rendering. In Proceedings of Symposium on Interactive 3D Graphics '90, pages 217-223, 1990.

[19] H. Löffelmann, L. Mroz, and E. Gröller. Hierarchical streamarrows for the visualization of dynamical systems. In Eurographics Workshop on Visualization in Scientific Computing '97, pages 155-164, 1997.

[20] L. Mroz, H. Hauser, and M. E. Gröller. Interactive highquality maximum intensity projection. In Proceedings of EUROGRAPHICS 'O0, pages 341-350, 2000.

[21] B. Pflesser, U. Tiede, and K. H. Höhne. Towards realistic visualization for surgery rehearsal. In Computer Vision, Virtual Reality and Robotics in Medicine, Proc. CVRMed '95, pages 487-491, 1995.

[22] P. Rheingans and D. Ebert. Volume illustration: Nonphotorealistic rendering of volume models. IEEE Transactions on Visualization and Computer Graphics, 7(3):253-264, 2001.

[23] B. Smits, J. Arvo, and D. Salesin. An importance-driven radiosity algorithm. In Proceedings of ACM SIGGRAPH '92, pages 273-282, 1992.

[24] M. Straka, M. Červeňanský, A. La Cruz, A. Köchl, M. Šrámek, Eduard Gröller, and Dominik Fleischmann. The VesselGlyph: Focus \& context visualization in CT-angiography. In Proceedings of IEEE Visualization '04 (to appear), 2004.

[25] L. Streit and J. Buchanan. Importance driven halftoning. In Proceedings of EUROGRAPHICS '98, pages 207-217, 1998.

[26] L. Szirmay-Kalos, B. Csébfalvi, and Werner Purgathofer. Importance driven quasi-random walk solution of the rendering equation. In Proceedings of WSCG '98, pages 379-385, 1998.

[27] S. Treavett and M. Chen. Pen-and-ink rendering in volume visualisation. In Proceedings of IEEE Visualization 'O0, pages 203-210, 2000.

[28] P. P. Vázquez, M. Feixas, M. Sbert, and W. Heidrich. Viewpoint selection using viewpoint entropy. In Proceedings of VMV '01, pages 273-280, 2001.

[29] I. Viola, A. Kanitsar, and M. E. Gröller. Importance-driven volume rendering. In Proceedings of IEEE Visualization'04, pages 139-145, 2004.

[30] J. Zhou, A. Döring, and K. Tönnies. Distance based enhancement for focal region based volume rendering. In Proceedings of Bildverarbeitung für die Medizin '04, pages 199-203, 2004. 


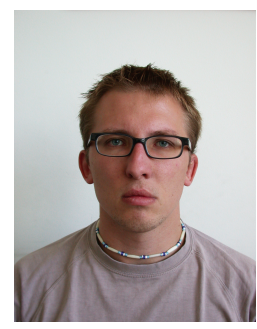

Ivan Viola graduated in 2002 from the Vienna University of Technology (TU Wien), Austria, as a Dipl.-Ing. (MSc) in the field of computer graphics and visualization. Since then he is a $\mathrm{PhD}$ student and research associate in the ADAPT research project in the field of medical visualization and real-time volume visualization(http: //www.cg.tuwien.ac.at/research/vis/adapt/). His main interests are efficient volume visualization in terms of quality, performance, and visual information.

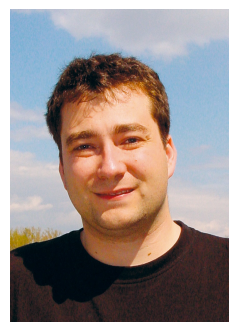

Armin Kanitsar is key developer in the 3D department of TIANI Medgraph and research associate at the Institute of Computer Graphics and Algorithms (ICGA), Vienna University of Technology. He received his $\mathrm{PhD}$ in 2004 for his thesis 'Curved Planar Reformation for Vessel Visualization' conducted at the ICGA. His main research interests include volume visualization, medical visualization, and image processing. Currently he is managing the research project ADAPT (http://www.cg.tuwien.ac.at/research/vis/adapt/), a close cooperation between TIANI Medgraph and the ICGA, with main focus on medical visualization. He acted as author or co-author for more than 20 international publications.

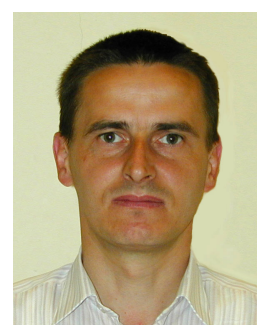

M . Eduard Gröller is associate professor at the Institute of Computer Graphics and Algorithms (ICGA), Vienna University of Technology. In 1993 he received his $\mathrm{PhD}$ from the same university. His research interests include computer graphics, flow visualization, volume visualization, and medical visualization. He is heading the visualization group at ICGA. The group performs basic and applied research projects in the area of scientific visualization (http://www. cg. tuwien.ac.at/research/vis/). Dr. Gröller has given lecture series on scientific visualization at various other universities (Tübingen, Graz, Praha, Bahia Blanca, Magdeburg). He is a scientific proponent and member of the Scientific Advisory Committee of the VRVis Kplus center of excellence (http: / / www.vrvis.at/) The center performs applied research in virtual reality and visualization. Dr. Gröller coauthored more than 100 scientific publications and acted as a reviewer for numerous conferences and journals in the field. $\mathrm{He}$ also serves on various program and paper committees. Examples include Computers\&Graphics, IEEE Transactions on Visualization and Graphics, VisSym, IEEE Visualization conference, Eurographics conference. He is head of the working group on computer graphics of the Austrian Computer Society and member of IEEE Computer Society, ACM (Association of Computing Machinery), GI (Gesellschaft für Informatik), OCG (Austrian Computer Society). 